\title{
Comparison of SPECT/CT, SPECT, and Planar Imaging with Single- and Dual-Phase 99mTc-Sestamibi Parathyroid Scintigraphy
}

\author{
William C. Lavely ${ }^{1}$, Sibyll Goetze ${ }^{1}$, Kent P. Friedman ${ }^{1}$, Jeffrey P. Leal ${ }^{1}$, Zhe Zhang ${ }^{2}$, Elizabeth Garret-Mayer ${ }^{2}$, \\ Alan P. Dackiw ${ }^{3}$, Ralph P. Tufano ${ }^{3}$, Martha A. Zeiger ${ }^{3}$, and Harvey A. Ziessman ${ }^{1}$ \\ ${ }^{I}$ Russell H. Morgan Department of Radiology and Radiological Sciences, Division of Nuclear Medicine, Johns Hopkins University, \\ Baltimore, Maryland; ${ }^{2}$ Department of Oncology and Biostatistics, Johns Hopkins University, Baltimore, Maryland; and ${ }^{3}$ Department of \\ Otolaryngology-Head and Neck Surgery, Johns Hopkins University, Baltimore, Maryland
}

\begin{abstract}
Various methodologies for ${ }^{99 m}$ Tc-sestamibi parathyroid scintigraphy are in clinical use. There are few direct comparisons between the different methods and even less evidence supporting the superiority of one over another. Some reports suggest that SPECT is superior to planar imaging. The addition of CT to SPECT may further improve parathyroid adenoma localization. The purpose of our investigation was to compare hybrid SPECT/CT, SPECT, and planar imaging and to determine whether dual-phase imaging is advantageous for the 3 methodologies. Methods: Scintigraphy was performed on 110 patients with primary hyperparathyroidism and no prior neck surgery. Of these, 98 had single adenomas and are the subject of this review. Planar imaging and SPECT/CT were performed at $15 \mathrm{~min}$ and $2 \mathrm{~h}$ after injection. Six image sets (early and delayed planar imaging, SPECT, and SPECT/CT) and combinations of the 2 image sets were reviewed for adenoma localization at 13 possible sites. Each review was scored for location and certainty of focus by 2 reviewer groups. Surgical location served as the standard. Sensitivity, specificity, area under the curve, positive predictive value, negative predictive value, and $\kappa$-values were determined for each method. Results: The overall $\kappa$-coefficient (certainty of adenoma focus) between reading groups was 0.68 ( $95 \%$ confidence interval, 0.66-0.70). The highest values were for dualphase studies that included SPECT/CT. Dual-phase planar imaging, SPECT, and SPECT/CT were statistically significantly superior to single-phase early or delayed imaging in sensitivity, area under the curve, and positive predictive value. Neither single-phase nor dual-phase SPECT was statistically superior to dual-phase planar imaging. Early-phase SPECT/CT in combination with any delayed imaging method was superior to dualphase planar imaging or SPECT for sensitivity, area under the curve, and positive predictive value. Conclusion: Early SPECT/ CT in combination with any delayed imaging method was statistically significantly superior to any single- or dual-phase planar or SPECT study for parathyroid adenoma localization. Localization with dual-phase acquisition was more accurate than with single-
\end{abstract}

Received Feb. 2, 2007; revision accepted Mar. 23, 2007.

For correspondence or reprints contact: Harvey A. Ziessman, MD, Johns Hopkins Outpatient Center, 601 N. Caroline St., JHOC Room 3231, Baltimore, MD 21287.

E-mail: hziessm1@jhmi.edu

COPYRIGHT @ 2007 by the Society of Nuclear Medicine, Inc. phase ${ }^{99 m} \mathrm{Tc}-$ sestamibi scintigraphy for planar imaging, SPECT, and SPECT/CT.

Key Words: hyperparathyroidism; parathyroid adenoma; parathyroid scintigraphy; SPECT/CT; minimally invasive parathyroidectomy

J Nucl Med 2007; 48:1084-1089

DOI: 10.2967/jnumed.107.040428

$\mathbf{P}$ rimary hyperparathyroidism is caused by the autonomous overproduction of parathyroid hormone. A single adenoma is found in $80 \%-90 \%$ of patients, double adenomas in 5\%-10\%, and 4-gland hyperplasia in $10 \%-15 \%(1)$. In the hands of an experienced surgeon, bilateral neck exploration has a success rate of greater than $95 \%(2,3)$. However, there has been a shift away from bilateral neck exploration with the advent of a more targeted surgical approach called minimally invasive parathyroidectomy $(4,5)$. With this approach, preoperative imaging and localization are critical to a successful operation.

Parathyroid scintigraphy has proven to be the single best imaging modality for preoperative localization of parathyroid adenomas, that is, superior to ultrasonography, CT, and MRI (6-8). The use of ${ }^{99 \mathrm{~m} T c-s e s t a m i b i}$ for parathyroid imaging was first reported in 1989 (9). This radiopharmaceutical has replaced ${ }^{201} \mathrm{Tl}$ because of its superior imaging characteristics and higher detection sensitivity $(7,10,11)$. However, different imaging methodologies have been used over the years. One approach uses thyroid imaging with ${ }^{99 \mathrm{~m} T c-p e r t e c h n e t a t e}$ or ${ }^{123}$ I-sodium iodide concomitantly and computer subtraction techniques. This approach requires injection of a second radiopharmaceutical; a cooperative, immobile patient; identical positioning for the 2 studies; and subtraction, with its potential artifacts (12-14).

In 1992, O'Doherty et al. described slower washout of $99 \mathrm{~m} T \mathrm{Tc}-\mathrm{sestamibi}$ from hyperfunctioning parathyroids than from the thyroid gland (10). Taillefer et al. demonstrated the diagnostic advantage of this differential washout and 
reported on the utility of a dual-phase methodology for parathyroid scintigraphy (15). This methodology requires 2 sets of images, one at 5-15 min (early) and one at 2-3 h (delayed). Investigations have reported good results $(11,16,17)$, similar in accuracy to the subtraction method, although few direct comparison studies have been reported (18-20).

Several investigations have reported improved sensitivity for the detection and localization of hyperfunctioning parathyroids with SPECT, compared with planar imaging (21-24), although 2 studies did not find SPECT superior to planar imaging $(19,20)$. Most studies have acquired only a single set of SPECT images, either early or delayed $(19,22,24,25) ; 2$ studies performed dual-phase SPECT $(26,27)$. The addition of anatomic imaging with CT software fusion (28) or hybrid SPECT/CT $(29,30)$ has the potential to improve preoperative localization. Investigations using hybrid imaging have been few, with limited numbers of patients studied and mixed results.

The purpose of this investigation was to directly compare the accuracy of ${ }^{99 \mathrm{~m}} \mathrm{Tc}$-sestamibi parathyroid SPECT/CT with SPECT and planar imaging for localization of parathyroid adenomas and to determine whether dual-phase imaging is advantageous over single-phase imaging for the 3 methodologies.

\section{MATERIALS AND METHODS}

\section{Patient Population}

Between April 2004 and July 2005, 259 consecutive patients with a diagnosis of hyperparathyroidism were referred for parathyroid scintigraphy. The entire imaging protocol was completed by 210 patients. Of these, 110 patients had undergone surgical exploration at our institution by the time of this review. Ninetyeight patients (30 male and 68 female), ranging in age from 28 to $94 \mathrm{y}$ (mean, $59.9 \mathrm{y}$ ), had a diagnosis of primary hyperparathyroidism and had single adenomas found at surgery. Four patients had double adenomas and 8 had hyperplasia. The 98 patients with single adenomas formed the basis of this investigation. This study was approved by the Johns Hopkins Medicine Institutional Review Board.

\section{Image Acquisition Protocol and Processing}

All patients were injected intravenously with $925-1,110 \mathrm{MBq}$ of ${ }^{99 \mathrm{~m}} \mathrm{Tc}$-sestamibi.

Planar Imaging Protocol. At $15 \mathrm{~min}$ and $2 \mathrm{~h}$ after injection, 10min anterior, $35^{\circ}$ right anterior oblique, and $35^{\circ}$ left anterior oblique planar images were acquired in a $128 \times 128$ matrix, with a $20 \%$ window centered around the $140-\mathrm{keV}$ photopeak using a lowenergy, high-resolution parallel collimator.

SPECT/CT Protocol. No oral or intravenous contrast material was used. Immediately after planar image acquisition, SPECT images were acquired on a Millennium VG/dual-head SPECT/CT system (GE Healthcare) or an Infinia Hawkeye SPECT/CT system (GE Healthcare). A step-and-shoot protocol of $25 \mathrm{~s} / 3^{\circ}$ for a total of 60 views per camera head was used. Immediately after SPECT acquisition, CT was performed. The parameters included a current of $2.5 \mathrm{~mA}$, a voltage of $140 \mathrm{kV}$, and $10-\mathrm{mm}$ slices reconstructed in a $256 \times 256$ matrix; the $\mathrm{CT}$ rotated at 2.6 rotations per minute. Acquisition required 7-9 min.
Transverse, coronal, and sagittal SPECT images were generated using a Hann prefilter and a Butterworth postprocessing filter with 2 ordered-subset expectation maximization (OSEM) iterations and a maximum of 10 OSEM subsets; the attenuation correction factor was 0.6. Transverse, coronal, and sagittal SPECT/CT images were generated using a Butterworth prefilter and postprocessing filter with 4 OSEM iterations for a maximum of 8 OSEM subsets.

\section{Image Interpretation and Scoring}

All planar and SPECT/CT images were masked as to patient identity and reviewed on a Xeleris workstation (GE Healthcare). Each study was read individually (early planar, delayed planar, early SPECT, delayed SPECT, early SPECT/CT, and delayed SPECT/CT) and also as paired image sets-for example, early planar with delayed planar, early planar with delayed SPECT, early planar with delayed SPECT/CT, early SPECT with delayed planar-for all possible combinations except early SPECT and early SPECT/CT and delayed SPECT and delayed SPECT/CT, because the SPECT is the same as that used for SPECT/CT. The result was a total of 19 different interpretations per patient (Table 1).

Masked image sets were interpreted by 2 groups of 2 readers who determined a consensus score for both groups for each location on all image sets or image set pairs. All readers were unaware of the laboratory, surgical, and pathologic results or of the reported interpretations. The image findings were scored for 13 different locations: right/left superior, right/left inferior, right/ left inferior-posterior, right/left intrathyroidal, right/left anterior mediastinum, right/left posterior mediastinum, and other. The score was dependent on the certainty of location and was graded from 1 to $5(1$ = very low, $2=$ low, $3=$ equivocal, $4=$ moderate, and $5=$ high). A score was also recorded on a worksheet for certainty of focus and was graded from 1 to $5(1=$ definitely negative, $2=$ probably negative, $3=$ equivocal, $4=$ probably positive, and $5=$ definitely positive). The results from each reading were recorded in an Excel (Microsoft) spreadsheet. The summed score of the 2 groups of reviewers was used for statistical analysis comparing the different methodologies for localization.

True location was determined by the surgeon. The mass of the parathyroid adenoma was obtained from pathologic reports.

\section{Statistical Analysis}

$\kappa$-statistics were calculated to measure agreement about the certainty of the parathyroid adenoma location between the 2 reader groups and were compared on the basis of asymptotically normal distribution-derived methods. Sensitivity (number of truepositives divided by number of true-positives plus false-negatives), specificity (number of true-negatives divided by number of truenegatives plus false-positives), positive predictive value (PPV; number of true-positives divided by number of true-positives plus false-positives), and negative predictive value (NPV; number of true-negatives divided by number of true-negatives plus falsenegatives) were calculated for localization, where a dichotomous call for certainty of location was defined as positive (grades 4 and 5) versus negative (grades 1-3). Surgical location served as the gold standard. A logistic regression approach was used to determine the area under the curve (AUC) for describing the accuracy of the imaging methodologies. Binomial testing was used to compare independent proportions, and the McNemar test was used to compare paired proportions. The variance of the AUC was constructed, and a $z$ score was computed for AUC comparisons. 
TABLE 1

Statistical Results for 19 Different Study Reads per Patient for Parathyroid Adenoma Localization in 98 Subjects

\begin{tabular}{lccccccc}
\hline \multicolumn{1}{c}{ Type } & Sensitivity (\%) & Specificity (\%) & AUC (\%) & PPV (\%) & NPV (\%) & к-coefficient & 90\% Cl \\
\hline Overall & 59.8 & 98.7 & 79.3 & 79.8 & 96.7 & 0.68 \\
Early planar & 34.0 & 99.0 & 66.5 & 74.7 & 94.7 & $0.66-0.70$ \\
Early SPECT & 53.5 & 98.7 & 76.1 & 77.0 & 96.2 & 0.61 & $0.38-0.63$ \\
Early SPECT/CT & 62.0 & 98.9 & 80.5 & 82.7 & 96.9 & 0.71 & $0.62-0.70$ \\
Delayed planar & 45.0 & 98.6 & 71.8 & 72.6 & 95.5 & 0.65 & $0.55-0.74$ \\
Delayed SPECT & 53.5 & 98.3 & 75.9 & 72.8 & 96.2 & 0.58 & $0.49-0.67$ \\
Delayed SPECT/CT & 53.5 & 98.1 & 75.8 & 70.4 & 96.2 & 0.67 & $0.59-0.76$ \\
Early planar-delayed SPECT & 57.5 & 98.8 & 78.1 & 79.9 & 96.5 & 0.67 & $0.58-0.76$ \\
Early planar-early SPECT/CT & 65.7 & 98.8 & 82.2 & 82.5 & 97.1 & 0.70 & $0.62-0.78$ \\
Delayed planar-delayed SPECT & 59.0 & 98.4 & 78.7 & 75.2 & 96.6 & 0.68 & $0.60-0.77$ \\
Delayed planar-delayed SPECT/CT & 62.5 & 98.5 & 80.5 & 78.1 & 96.9 & 0.69 & $0.61-0.77$ \\
Early planar-delayed planar & 56.5 & 98.7 & 77.6 & 79.0 & 96.4 & 0.70 & $0.61-0.78$ \\
Early planar-delayed SPECT & 63.5 & 98.6 & 81.1 & 79.4 & 97.0 & 0.71 & $0.63-0.79$ \\
Early planar-delayed SPECT/CT & 61.0 & 98.4 & 79.7 & 76.7 & 96.8 & 0.57 & $0.48-0.66$ \\
Early SPECT-delayed planar & 64.5 & 98.9 & 81.7 & 82.7 & 97.1 & 0.71 & $0.63-0.79$ \\
Early SPECT-delayed SPECT & 61.5 & 98.7 & 80.1 & 79.4 & 96.8 & 0.67 & $0.58-0.75$ \\
Early SPECT-delayed SPECT/CT & 66.0 & 98.8 & 82.4 & 82.5 & 97.2 & 0.66 & $0.58-0.75$ \\
Early SPECT/CT-delayed planar & 72.5 & 99.0 & 85.8 & 86.3 & 97.7 & 0.77 & $0.69-0.84$ \\
Early SPECT/CT-delayed SPECT & 73.0 & 99.4 & 86.2 & 90.7 & 97.8 & 0.79 & $0.72-0.86$ \\
Early SPECT/CT-delayed SPECT/CT & 72.0 & 99.1 & 85.6 & 87.3 & 97.7 & 0.76 & $0.69-0.83$ \\
\hline
\end{tabular}

$P$ values were adjusted for multiple-hypothesis testing by controlling a false-discovery rate of $5 \%$ using the Benjamini-Hochberg false-discovery-rate method (31). All statistical tests were 2-sided and considered to be statistically significant at a $P$ value of 0.05 or less. Analyses were conducted with SAS software (version 9.1; SAS Institute Inc.) and the R statistical package (version 2.3.1).

\section{RESULTS}

Of the 98 single adenomas, 8 were right superior, 15 were left superior, 24 were right inferior, 25 were left inferior, 17 were right inferior-posterior, 6 were left inferiorposterior, 1 was right intrathyroidal, 1 was left intrathyroidal, and 1 was mediastinal.

\section{Agreement Between Readers}

The overall $\kappa$-coefficient for agreement for parathyroid adenoma location between the 2 reading groups for the 19 patient study readings was 0.68 (95\% CI, 0.66-0.70) (Table $1)$. The highest $\kappa$-values were for dual-phase studies that included early SPECT/CT (0.76-0.79), followed by dualphase studies that included early SPECT (0.66-0.71). The lowest $\kappa$-values were for single-phase studies (0.50-0.61).

Correlation of certainty of location with several covariates revealed a significant correlation for adenoma weight $(P<0.0001)$, age $(P<0.0001)$, and PTH level $(P<$ $0.0001)$ but not for race, serum calcium level, or sex.

\section{Localization}

Sensitivity, specificity, PPV, and NPV for localization were derived from the 19 image set readings for each patient and are listed in Table 1. $P$ values denoting statistical significance for PPV, sensitivity, and AUC for different comparisons are listed in Table 2.
Overall sensitivity for localization for all modalities was $60 \%$, specificity was $99 \%$, PPV was $80 \%$, NPV was $97 \%$, and AUC was $80 \%$. Sensitivity ranged from $34 \%$ to $45 \%$ for single-phase early or delayed planar images to $73 \%$ for the dual-phase studies that included early SPECT/CT. PPV ranged from $70 \%$ to $73 \%$ for single-phase delayed planar, SPECT, or SPECT/CT studies to $86 \%-91 \%$ for dual-phase studies that included early SPECT/CT. AUC ranged from $67 \%$ to $76 \%$ for single-phase planar, SPECT, or SPECT/CT studies to $86 \%$ for dual-phase studies that included early SPECT/CT.

All specificity values were greater than $98.0 \%$, and values for NPV were equal to or greater than $95 \%$. These high values were biased by methodology. Any of the 13 possible locations not graded positive were considered negative. Thus, a patient with correct localization of a single adenoma by scintigraphy had one true-positive and 12 truenegative locations.

The following sections summarize the most important statistical comparisons for localization of parathyroid adenomas. More comparisons are shown in Table 2.

Planar Imaging. Dual-phase planar imaging had significantly higher sensitivity $(P<0.0001, P=0.001)$ and AUC $(P<0.0001, P=0.002)$ than did early or delayed planar imaging alone.

SPECT. Single-phase early SPECT was not significantly different from delayed SPECT for sensitivity, AUC, or PPV. Dual-phase SPECT had a greater sensitivity $(P=0.056$, $P=0.039)$ and AUC $(P<0.045, P<0.049)$ for localization than did single-phase early or delayed SPECT alone.

SPECT vs. Planar Imaging. Although there was a trend for higher localization sensitivity and AUC for SPECT than for planar imaging, neither single-phase nor dual-phase 
TABLE 2

$P$ Values for PPV, Sensitivity, and AUC for Comparisons of Early and Delayed Planar, SPECT, and SPECT/CT Imaging Methods

\begin{tabular}{|c|c|c|c|}
\hline \multirow[b]{2}{*}{ Imaging method } & \multicolumn{3}{|c|}{$P$} \\
\hline & PPV & Sensitivity & AUC \\
\hline Planar E vs. planar D & 0.09 & 0.045 & 0.014 \\
\hline Planar E/D vs. planar E or planar D & NS & $<0.0001$ & 0.002 \\
\hline SPECT E vs. SPECT D & NS & NS & NS \\
\hline SPECT E/D vs. SPECT E or SPECT D & NS & 0.056 & 0.045 \\
\hline SPECT E vs. planar E & NS & $<0.0001$ & $<0.0001$ \\
\hline SPECT D vs. planar D & NS & 0.056 & 0.023 \\
\hline SPECT E or SPECT D vs. planar E/D & NS & NS & NS \\
\hline SPECT E/D vs. planar E/D & NS & NS & NS \\
\hline SPECT/CT E vs. SPECT/CT D & 0.027 & 0.076 & 0.036 \\
\hline SPECT/CT E/D vs. SPECT/CT E & NS & 0.003 & 0.001 \\
\hline SPECT/CT E/D vs. SPECT/CT D & 0.001 & $<0.0001$ & $<0.0001$ \\
\hline SPECT/CT E/D vs. SPECT/CT E/SPECT D & NS & NS & NS \\
\hline SPECT/CT E/D vs. SPECT/CT E/planar D & NS & NS & NS \\
\hline SPECT/CT E or SPECT/CT D vs. planar E/D & NS & NS & NS \\
\hline SPECT/CT E/D vs. planar E/D & 0.05 & $<0.0001$ & $<0.0001$ \\
\hline SPECT/CT E/planar D vs. planar E/D & NS & $<0.0001$ & $<0.0001$ \\
\hline SPECT/CT E vs. SPECT E & NS & NS & 0.049 \\
\hline SPECT/CT E/D vs. SPECT E/D & NS & 0.011 & 0.004 \\
\hline SPECT/CT E/SPECT D vs. SPECT E/D & 0.011 & 0.003 & 0.001 \\
\hline
\end{tabular}

SPECT had significantly higher AUC, sensitivity, or PPV than did dual-phase planar imaging.

SPECT/CT. Early SPECT/CT had higher sensitivity $(P=$ 0.076), AUC $(P=0.036)$, and PPV $(P=0.027)$ than did delayed SPECT/CT for localization. Dual-phase SPECT/ CT had significantly higher sensitivity $(P=0.003, P<$ $0.0001)$ and AUC $(P=0.001, P<0.0001)$ than did early or delayed single-phase SPECT/CT alone. There was no statistically significant difference between the 3 image sets that included early SPECT/CT when combined with any delayed phase-that is, planar imaging, SPECT, or SPECT/CT.

SPECT/CT vs. Planar Imaging. Single-phase SPECT/CT (early or delayed) was not significantly superior to dualphase planar imaging for sensitivity, AUC, or PPV of localization. Dual-phase SPECT/CT had significantly higher sensitivity $(P<0.0001)$, AUC $(P<0.0001)$, and PPV $(P=0.05)$ than did dual-phase planar imaging. Early SPECT/CT in combination with delayed planar imaging had higher sensitivity $(P<0.0001)$ and AUC $(P<0.0001)$ than did dual-phase planar imaging.

SPECT/CT vs. SPECT. Dual-phase SPECT/CT was statistically superior to dual-phase SPECT for sensitivity $(P=$ $0.011)$ and $\operatorname{AUC}(P=0.004)$ for localization. Early SPECT/CT with delayed SPECT was significantly superior to dual-phase SPECT for sensitivity $(P=0.003)$, AUC $(P=0.001)$, and PPV $(P=0.011)$. When inferior and inferior-posterior locations were combined, sensitivity, AUC, and PPV increased for all 19 imaging methods.
Overall PPV increased from $80 \%$ to $92 \%$. Overall AUC increased from $79 \%$ to $84 \%$. Overall sensitivity increased from $60 \%$ to $69 \%$. There was little change in NPV $(97 \%-$ $97 \%$ ) or specificity (98\%-99\%). However, the statistical advantage of SPECT/CT was no longer apparent.

\section{DISCUSSION}

Publications over the years have described many different scintigraphic methodologies for the preoperative localization of hyperfunctioning parathyroid glands. The optimal methodology for ${ }^{99 \mathrm{~m}}$ Tc-sestamibi parathyroid scintigraphy was not clear from a review of the medical literature because few investigators have directly compared the accuracy of different methodologies. Even when methods have been compared, the statistical advantage of one method over another has rarely, if ever, been convincingly demonstrated. As a result, many different methodologies are in clinical use today. Our investigation has directly compared many commonly used methodologies, and we report statistically significance differences in localization sensitivity, AUC, and PPV.

For successful minimally invasive parathyroid surgery, accurate preoperative localization is mandatory. Because of their embryologic descent, superior parathyroid glands tend to be more posterior than inferior glands and are often found in the tracheoesophageal groove. Surgery for an inferior-posterior gland (superior gland) is often more complex because it lies in intimate association with the 
recurrent laryngeal nerve. Preoperative knowledge of the likelihood of an inferior-posterior location facilitates exploration. Thus, SPECT is theoretically advantageous over planar imaging because it provides details of the anterior or posterior adenoma location.

For various radiopharmaceuticals and different types of scintigraphic studies, the superior contrast resolution of SPECT, compared with that of planar imaging, has often translated into increased lesion detectability. For parathyroid scintigraphy, several studies have reported higher sensitivity for SPECT than for planar imaging-from $11 \%$ to $18 \%$ higher-although no study has reported a statistically significant difference (21-24). Two studies reported no advantage for SPECT over planar imaging $(19,20)$. Although our data showed a trend toward higher sensitivity, AUC, and PPV for SPECT than for planar imaging, the difference was not statistically significant.

SPECT is often acquired at a single time interval, either early or delayed. With early SPECT, one investigational group has reported a high sensitivity $(96 \%)(22,24)$. Using delayed SPECT, one large study reported good sensitivity (87\%) (25). However, another report found poorer results (78\%) (19). Only one prior investigation directly compared early and delayed SPECT (26); these authors found that early SPECT had higher sensitivity $(91 \%)$ than delayed SPECT (74\%), although no statistically significant difference was reported. Our data showed a trend toward superior sensitivity and AUC for parathyroid adenoma localization for early SPECT than for delayed SPECT; however, this trend did not reach statistical significance. Dual-phase SPECT was statistically significantly superior to singlephase SPECT.

Prior publications have often reported their results as sensitivity for detection of an adenoma in a patient with hyperparathyroidism or, in other cases, correct lateralization. However, with minimally invasive surgery, exact preoperative localization becomes paramount and is the reason we emphasize the sensitivity, AUC, and PPV of localization.

Software fusion of separately acquired parathyroid SPECT and CT images has been reported to improve the accuracy of localization over that with SPECT alone in small numbers of patients (28). Hybrid SPECT/CT has the advantage of sequentially imaging the patient in the same position on the same imaging table. The hybrid system applied in this investigation used a $2.5-\mathrm{mA}$ low-resolution single-slice CT scanner, which limited exact anatomic localization in many cases. The radiation dose to the patient from the $2 \mathrm{CT}$ scans with dual-phase imaging was considerably less than that from a typical single diagnostic CT scan.

Two published investigations on the value of hybrid SPECT/CT for parathyroid scintigraphy, with smaller patient populations than ours, reported differing results. In one study of 48 patients, early SPECT in combination with dual-phase planar imaging identified $89 \%$ of surgically confirmed parathyroid adenomas, with or without CT fusion. The investigators concluded that $\mathrm{CT}$ fusion added no value to SPECT alone (29). In another investigation of 36 patients, in which SPECT/CT was performed only on patients with negative or diagnostically uncertain planar imaging results, SPECT/CT contributed to localization and surgical planning in $39 \%$ of patients (30). In both studies, early SPECT/CT was compared with dual-phase planar imaging.

In our investigation, early SPECT/CT alone was not significantly better than dual-phase planar imaging for parathyroid adenoma localization. However, dual-phase SPECT/CT was statistically significantly superior to singlephase SPECT/CT and superior to dual-phase planar imaging and dual-phase SPECT. Early SPECT/CT in combination with delayed SPECT or delayed planar imaging was also statistically superior to dual-phase planar imaging or SPECT.

A major advantage of SPECT/CT seems to be its ability to differentiate inferior from inferior-posterior glands (superior glands in the tracheoesophageal groove). The statistical advantage of SPECT/CT was not evident when analysis was done with the inferior and inferior-posterior locations combined.

\section{CONCLUSION}

Hybrid SPECT/CT combines the 3-dimensional functional information of SPECT with the anatomic information of $\mathrm{CT}$, thereby improving preoperative localization. Early SPECT/CT in combination with any delayed imaging method (SPECT/CT, SPECT, or planar imaging) was statistically superior to dual-phase planar imaging or dualphase SPECT. For all 3 methodologies (planar imaging, SPECT, and SPECT/CT), dual-phase imaging was superior to single-phase imaging. These findings strongly suggest that dual-phase imaging, with early SPECT/CT whenever possible, should be part of the routine preoperative evaluation of patients with primary hyperparathyroidism in this new age of minimally invasive parathyroidectomy.

\section{REFERENCES}

1. Ruda JM, Hollenbeak C, Stack BC Jr. A systematic review of the diagnosis and treatment of primary hyperparathyroidism from 1995 to 2003. Otolaryngol Head Neck Surg. 2005;132:359-372.

2. Satava RM, Beahrs OH, Scholz DA. Success rate of cervical exploration for hyperparathyroidism. Arch Surg. 1975;110:625-627.

3. Rose MD, Wood TF, Van Herele, et al. Long term management and outcome of parathyroid-ectomy for sporadic primary multiple gland disease. Arch Surg. 2001;136:621-626.

4. Grant CS, Thompson G, Farley D, van Heerden J. Primary hyperparathyroidism surgical management since the introduction of minimally invasive parathyroidectomy: Mayo clinic experience. Arch Surg. 2005;140:472-479.

5. Mekel M, Mahajna A, Ish-Shalom S, et al. Minimally invasive surgery for treatment of hyperparathyroidism. Isr Med Assoc J. 2005;7:323-327.

6. Ishibashi M, Nishida H, Hiromoatsu Y, et al. Comparison of technetium-99mMIBI, technetium-99 tetrofosmin, ultrasound, and MRI for localization of abnormal parathyroid glands. J Nucl Med. 1998;39:320-324.

7. Geatti O, Shapiro B, Orsolon P, et al. Localization of parathyroid enlargement: experience with technetium-99m methoxyisobutylisonitrile and thallium-201 scintigraphy, ultrasound and computed tomography. Eur J Nucl Med. 1994;21: $17-23$. 
8. Peeler BB, Martin WH, Sandler MP, Goldstein RE. Sestamibi parathyroid scanning and preoperative localization studies for patients with recurrent/ persistent hyperparathyroidism or significant comorbid conditions: development of an optimal localization strategy. Am Surg. 1997;63:37-46.

9. Coakley AJ, Kettle AG, Wells CP, et al. 99Tcm sestamibi: a new agent for parathyroid imaging. Nucl Med Commun. 1989;10:791-794.

10. O'Doherty MJ, Kettle AG, Wells P, et al. Parathyroid imaging with technetium99m-sestamibi: preoperative localization and tissue uptake studies. J Nucl Med. 1992;33:313-319.

11. Rauth J, Sessions RB, Shupe SC, Ziessman HA. Comparison of Tc-99m MIBI and Tl-201/Tc-99m pertechnetate for diagnosis of primary hyperparathyroidism. Clin Nucl Med. 1996;21:602-608.

12. Hindie E, Melliere D, Perlemuter L, et al. Primary hyperparathyroidism: higher success rate of first surgery after preoperative Tc-99m sestamibi-I-123 subtraction scanning. Radiology. 1997;204:221-228.

13. Thule P, Thakore K, Vansant J, et al. Preoperative localization of parathyroid tissue with Tc-99m sestamibi/I-123 subtraction scanning. J Clin Endocrinol Metab. 1994;78:77-82.

14. Wei JP, Burke GJ, Mansberger AR Jr. Preoperative imaging of abnormal parathyroid glands in patients with hyperparathyroid disease using combination Tc-99m-pertechnetate and Tc-99m-sestamibi radionuclide scans. Ann Surg. 1994; 219:568-572.

15. Taillefer R, Boucher Y, Potvin C, Lambert R. Detection and localization of parathyroid adenomas in patients with hyperparathyroidism using a single radionuclide imaging procedure with technetium-99m-sestamibi (double-phase study). J Nucl Med. 1992;33:1801-1807.

16. Blanco I, Carril JM, Bazo I, et al. Double-phase Tc-99m sestamibi scintigraphy in the preoperative location of lesions causing hyperparathyroidism. Clin Nucl Med. 1998;23:291-297.

17. Irvin GL III, Sfakianakis G, Yeung L, et al. Ambulatory parathyroidectomy for primary hyperparathyroidism. Arch Surg. 1996;131:1074-1078.

18. Leslie WD, Dupont JO, Bybel B, Riese KT. Parathyroid ${ }^{99 m} \mathrm{Tc}$-sestamibi scintigraphy: dual tracer subtraction is superior to double phase washout. Eur $J$ Nucl Med Mol Imaging. 2002;29:1566-1570.

19. Chen CC, Holder LE, Scovill WA, et al. Comparison of parathyroid imaging with technetium-99m-pertechnetate/sestamibi subtraction, double phase technetium-99m-sestamibi and technetium-99m-sestamibi SPECT. J Nucl Med. 1997;38:834-839.

20. Staudenherz A, Abela C, Niederle B, et al. Comparison and histopathological correlation of three parathyroid imaging methods in a population with a high prevalence of concomitant thyroid disease. Eur J Nucl Med. 1997;24:143-149.

21. Moka D, Voth E, Dietlein M, et al. Technetium 99m MIBI-SPECT: a highly sensitive diagnostic tool for localization of parathyroid adenomas. Surgery. 2000;128:29-35.

22. Schachter PP, Issa N, Shimonov M, Czerniak A, Lorberboym M. Early, postinjection MIBI-SPECT as the only preoperative localizing study for minimally invasive parathyroidectomy. Arch Surg. 2004;139:433-437.

23. Slater A, Gleeson FV. Increased sensitivity and confidence of SPECT over planar imaging in dual-phase sestamibi for parathyroid adenoma detection. Clin Nucl Med. 2005;30:1-3.

24. Lorberboym M, Minski I, Macadziob S, et al. Incremental diagnostic value of preoperative ${ }^{99 \mathrm{~m}} \mathrm{Tc}-\mathrm{MIBI}$ SPECT in patients with a parathyroid adenoma. $\mathrm{J} \mathrm{Nucl}$ Med. 2003;44:904-908.

25. Civelek AC, Ozalp E, Donovan P, Udelsman R. Prospective evaluation of delayed technetium-99m sestamibi SPECT scintigraphy for preoperative localization of primary hyperparathyroidism. Surgery. 2002;131:149-157.

26. Perez-Monte JE, Brown ML, Shah AN, et al. Parathyroid adenomas: accurate detection and localization with Tc-99m sestamibi SPECT. Radiology. 1996;201: 85-91.

27. Sfakianakis GN, Irvin GL, Foss J, et al. Efficient parathyroidectomy guided by SPECT-MIBI and hormonal measurements. J Nucl Med. 1996;37:798-804.

28. Profanter C, Wetscher GJ, Gabriel M, et al. CT-MIBI image fusion: a new preoperative localization technique for primary, recurrent, and persistent hyperparathyroidism. Surgery. 2004;135:157-162.

29. Gayed IW, Kim EE, Broussard WF, et al. The value of $99 \mathrm{~m}-\mathrm{Tc}$-sestamibi SPECT/CT over conventional SPECT in the evaluation of parathyroid adenomas and hyperplasia. J Nucl Med. 2005;46:248-252.

30. Krausz Y, Bettman L, Guralnik L, et al. Technetium-99m-MIBI SPECT/CT in primary hyperparathyroidism. World J Surg. 2006;30:76-83.

31. Benjamini Y, Hochberg Y. Controlling the false discovery rate: a practical and powerful approach to multiple testing. J Roy Statist Soc B. 1995;57: 289-300. 